diagnosis of obesity in 2005-2006 and 2009-2010 are 7.14\% and $15.63 \%$, respectively. The admission rates with obesity have increased significantly in 2009-2010 compared to 2005-2006 with an OR of 2.52 (1.14 to 5.08) with a p-value of 0.021. Average length of stay in 2005-2006 is 20.6 days and in 2009-2010 19.9 days respectively. Average length of stay in non-obese patient during the same period is 8.9 days. There are a higher proportion of obese women as compared to men admitted to NIV unit in 2009-2010. Abstract P152 Table 1.

Abstract P152 Table 1

\begin{tabular}{lll}
\hline & April 2005 to March 2006 & April 2009 to March 2010 \\
\hline Total admissions & 154 & 160 \\
Obese patients & 11 & 25 \\
Length of stay & 20.6 days & 19.9 days \\
Average weight & $121.33 \mathrm{~kg}$ & $114.34 \mathrm{~kg}$ \\
Average BMI & 42.11 & 43.03 \\
\hline
\end{tabular}

Conclusion There is a significant increase in number of admissions who are obese to the NIV unit. This is consistent with the observation that there is an increase in the BMI of acute general admissions. These cohorts of obese patients have a higher average length of stay and consume enormous amount of healthcare resources. Interventions to reduce obesity in the general public need to be taken up as a priority to preserve limited healthcare resources.

\section{P153 THE EFFECT OF BODY MASS INDEX ON OUTCOMES OF ACUTE NON-INVASIVE VENTILATION (NIV) IN A DISTRICT GENERAL HOSPITAL}

doi:10.1136/thx.2010.151043.4

H Liddicoat, C Zammitt, J Loft, I Moonsie, H Makker. North Middlesex Hospital, London, UK

Introduction The increasing prevalence of obesity is likely to affect the caseload of Non-invasive Ventilation (NIV) units. We hypothesised that outcomes in the obese and morbidly obese population would differ from patients with a normal Body Mass Index (BMI) on the NIV unit.

Method We audited all patients admitted to the NIV unit from January 2007 to December 2009. Patient demographics, admitting diagnosis, baseline arterial blood gases (ABG), duration on NIV, length of stay and outcome were recorded on BTS NIV audit proformas. BMI was obtained from GP records. Data were analysed using Prism 5

Results A total of 176 patients (46\% male, median age 74), were managed on the NIV unit. The admitting diagnosis was COPD $(59 \%)$, Obesity hypoventilation syndrome (OHS) (13\%), Cardiac failure $(13 \%)$, Neuromuscular disorders $(2 \%)$ or "other" diagnoses (13\%). The median BMI was 27 (IOR 22.7-35.0); 6\% of patients were underweight, $33 \%$ normal weight, $18 \%$ overweight, $26 \%$ obese and $17 \%$ morbidly obese. An increasing proportion of females were found in the obese and morbidly obese population $\left(\chi^{2} p=0.003\right)$. No statistical differences were found in baseline ABG and NIV duration between different BMI categories. Compared to patients with a normal weight, obese and morbidly obese individuals had significantly longer lengths of hospital stay (Mann-Whitney $U$ test $p=0.004$ and $p=0.001$, respectively). Outcomes did not differ between normal weight, underweight, or overweight patients. However, obese and morbidly obese patients were significantly more likely to survive admission compared to patients with a normal BMI (Abstract P153 Figure 1; $\chi^{2} p=0.035$ and $p=0.0008$, respectively).

\section{Outcome of Patients Admitted to Acute NIV Unit}

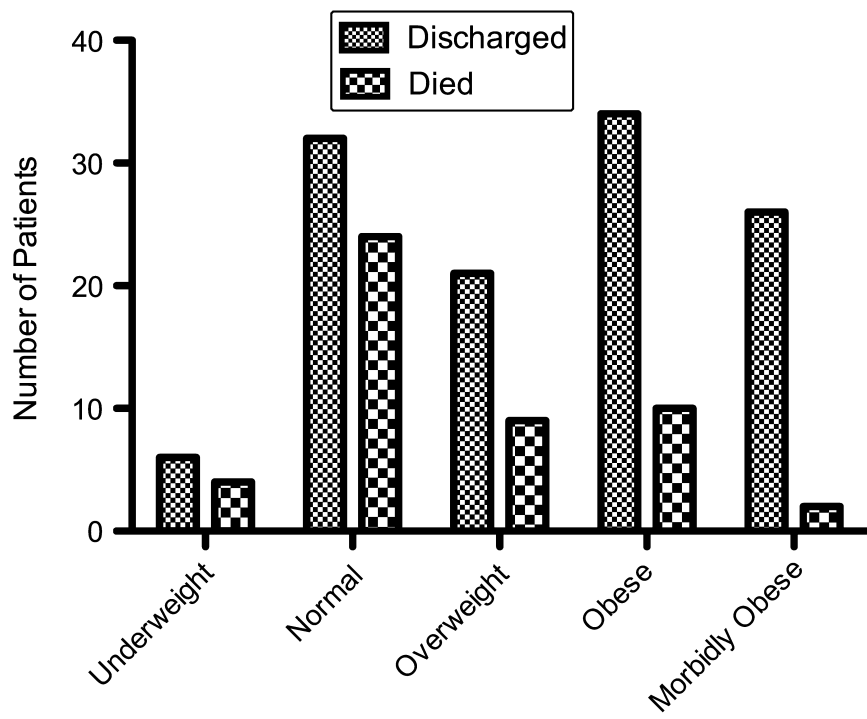

BMI Category

Abstract P153 Figure 1 Outcome of patients admitted to NIV Unit.

Similarly, there was no significant difference in age, gender, ABG parameters, NIV duration or length of stay between COPD and $\mathrm{OHS}$ patients. However, OHS patients were significantly more likely to survive admission $\left(\chi^{2} \mathrm{p}=0.0042\right)$.

Conclusion The outcomes for obese and morbidly obese patients were significantly better than patients with a normal BMI, although the length of stay was higher. Despite similar demographic features and metabolic disturbance of COPD and OHS patients, OHS patients were more likely to survive admission.

\section{P154 THE PATIENTS' EXPERIENCE OF NIV. A PHENOMENOLOGICAL STUDY}

doi:10.1136/thx.2010.151043.5

H Matthews. James Paget NHS University Foundation Trust, Gt Yarmouth, UK

Background The Evidence that NIV is an effective treatment for patients with COPD who present in Acute Type 2 Respiratory Failure is well documented. Yet despite NIV being considered the gold standard for treating patients with this life threatening condition, no published research could be found on how individuals experienced NIV in the acute setting. The Respiratory Team has led the NIV service for the last 7 years and our knowledge has grown with every patient we have treated with NIV. However during the follow-up of patients who had undergone NIV it became apparent that some of them had strange tales to tell. They told of dreams and images that they associated with their episode on NIV. Some refused having NIV again due to their dislike of the treatment. This was not something which had been discussed whilst in hospital and warranted investigation.

The Study This phenomenological study aimed to explore the experiences of 8 patients with COPD who underwent NIV for Acute Type 2 RF. To gain access to the rich data, in-depth unstructured interviews were conducted. The data were analysed using a framework by Deikelman.

The Outcomes Some themes which emerged from the data were expected however, the main theme of delirium was not. This unpredicted finding raised issues about mental capacity and decision making. This new understanding of delirium and its possible 
consequences has far reaching implications for how we care for this vulnerable group especially when it comes to nursing them on ward areas rather than the HDU setting. The findings from this study have proved fascinating and set the scene for further exploration.

\section{P155 NON-INVASIVE VENTILATION (NIV) IN ACUTE HYPERCAPNIC RESPIRATORY FAILURE IN RESTRICTIVE LUNG DISEASES (RLD)}

doi:10.1136/thx.2010.151043.6

${ }^{1} \mathrm{~A}$ Thomas, ${ }^{1} \mathrm{~A}$ Ali, ${ }^{2} \mathrm{~B}$ Chakraborty, ${ }^{1} \mathrm{~B}$ Beauchamp, ${ }^{1} \mathrm{E}$ Gallagher, ${ }^{1} \mathrm{D}$ Banerjee, ${ }^{1} \mathrm{R}$ Mukherjee. ${ }^{1}$ Heart of England NHS Foundation Trust, Birmingham, UK; ${ }^{2}$ School of Mathematics, University of Birmingham, Birmingham, UK

Introduction Successful NIV has been described in RLD (thoracic cage disease, obesity-hypoventilation and neuromuscular diseases) and given the success in chronic ventilatory failure, NIV should be considered as the treatment of choice in decompensated ventilatory failure due to RLD in an acute setting.

Methods Analysis of initial ABG in those admitted to a NIV unit on a respiratory ward with a diagnosis of RLD, requiring NIV for an acute episode of respiratory failure $\left(\mathrm{pH}<7.35\right.$ and $\left.\mathrm{pCO}_{2}>6.0 \mathrm{kPa}\right)$ admitted between 01 August 2004 and 31 December 2009. Patients included were those with respiratory failure as a consequence of RLD. Those who were either admitted or managed in HDU were excluded from the analysis. The admission episodes were stratified by initial $\mathrm{pH}$ ranges (predictor variable) and in-hospital mortality was recorded (outcome variable).

Results In 270 admissions (221 unique patients) with RLD requiring NIV for acute respiratory failure, the overall mortality was 35 (13.0\%). There was a significant increase in mortality in the group where ABG $\mathrm{pH}<7.15$ (40.9\%). The difference is significant when compared to the group with a $\mathrm{pH}$ between 7.15 and $7.26(9.6 \%)(\mathrm{p}=0.027)$. There was no significant difference in mortality in the group with $\mathrm{pH} 7.15$ to 7.26 to the group with $\mathrm{pH} 7.26$ to 7.35 ( $\mathrm{p}=0.94$ ): See Abstract P155 Figure 1 . The plot of fitted logistic regression equation with initial $\mathrm{pH}$ (the dotted lines indicate $95 \%$ confidence band).

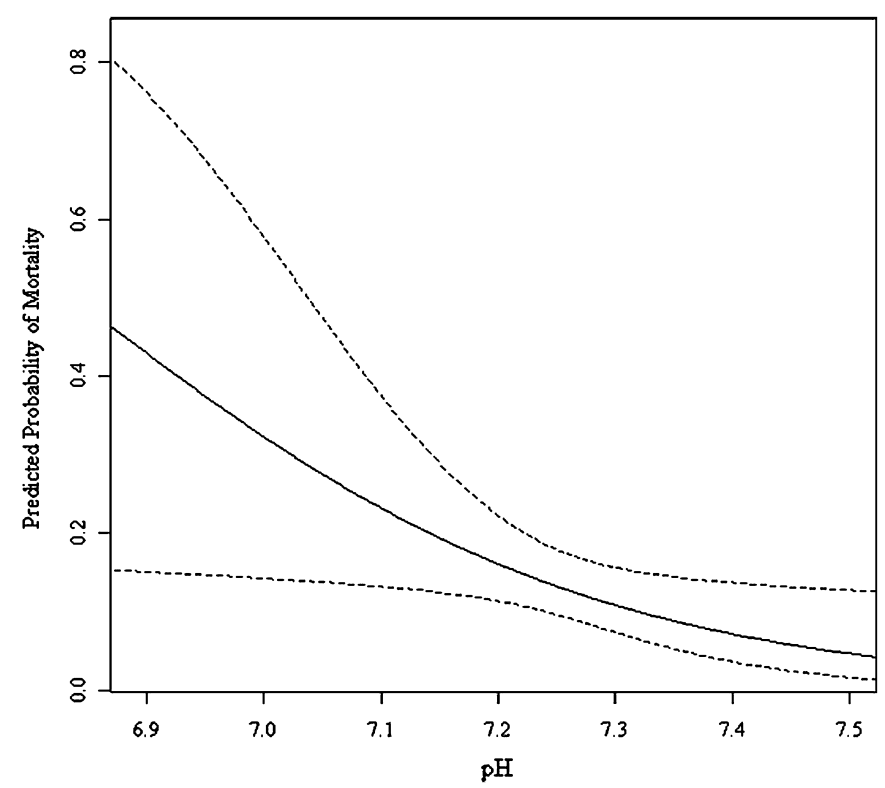

Abstract P155 Figure 1 The plot of fitted logistic regression equation with initial $\mathrm{pH}$ (the dotted lines indicate 95\% confidence band).

Conclusion For those patients with RLD treated with NIV on a respiratory ward, the mortality increases with the severity of acidosis. As no current guidelines indicate a $\mathrm{pH}$ cut-off for the ward based management of RLD with NIV, from our results we propose that this could be an initial ABG pH of 7.15.

\section{P156 DOES ANALYSIS OF PATIENT-VENTILATOR INTERACTION OFFER BENEFITS IN ADDITION TO OVERNIGHT PULSE OXIMETRY IN PATIENTS WITH MOTOR NEURONE DISEASE BEING FOLLOWED ON NON-INVASIVE VENTILATION?}

doi:10.1136/thx.2010.151043.7

${ }^{1} \mathrm{~B}$ Chakrabarti, ${ }^{2} \mathrm{H}$ Ando, ${ }^{3} \mathrm{E}$ Thornton, ${ }^{4} \mathrm{~V}$ Ford, ${ }^{2} \mathrm{C}$ A Young, ${ }^{1} \mathrm{R} \mathrm{M}$ Angus. ${ }^{1}$ Aintree Chest Centre, University Hospital Aintree, Liverpool, UK; ${ }^{2}$ Walton Centre for Neurology and Neurosurgery, Liverpool, UK; ${ }^{3}$ School of Psychology, University of Liverpool, Liverpool, UK; ${ }^{4}$ Department of Physiotherapy, University Hospital Aintree, Liverpool, UK

Introduction Non-invasiveventilation (NIV) is increasingly being offered to Motor Neurone Disease (MND) patients as studies report benefit insurvival and quality of life (QoL). Ventilator technology allows monitoring of patient-ventilator interaction. It is less clear whether such data yield useful additional information over pulse overnight oximetry. In a longitudinal study, MND patients were assessed both physiologically and psychologically prior to NIV initiation and 3monthly until death.

Methods 35 patients were enrolled; 28 offered NIV; 11 declined and 17 established on treatment. At initiation all had nocturnal hypoventilation by symptoms andoximetry criteria. Nine patients ( 8 male; median age 60 years) had $\geq 12$ months follow-up on ventilation; none required oxygen with NIV. Patient-ventilator interaction was assessed by analysis of the 'memory card' from the ventilator at 4-6 months post initiation (Point A) and at 10-12 months (Point B); QoL assessed by validated questionnaires within 2 weeks of memory card analysis.

Results Median overnight oxygen saturation in the sample was $93 \%$ at point $\mathrm{A}$ and $94 \%$ at $\mathrm{B}$; only 3 and 2 patients respectively exhibited 'sub-optimal' oximetry that is, >30 min below $90 \%$ saturation resulting in adjustment of ventilation. Minute Ventilation (MV) fell in the group from Point A to B (mean 6.97-6.34 1/min); a fall in MV (noted in five patients of which 1 exhibited "sub-optimal" oximetry) was associated with a fall in ALS FRS score denoting worsening health status (correlation coefficient $0.73 ; p=0.026$ ). Ventilator triggering decreased overall in the group from Points A to B (mean 69.95-61.57\% proportion of triggered breaths); a decrease in ventilator triggering (noted in six patients of which 1 exhibited "suboptimal" oximetry) correlated with an increase in ALS AQ domain for emotion denoting worsening emotional functioning (correlation coefficient -0.89; $\mathrm{p}=0.002$ ) with anon-significant trend noted between fall in triggered breaths and increase in Hospital Anxiety and Depression (HAD) score (correlation coefficient -0.68 ; $\mathrm{p}=0.055$ ).

Conclusion In MND, monitoring of patient-ventilator interaction may serve as a useful adjunct to pulse oximetry and symptom assessment. Further studies are needed to ascertain whether adjustment of ventilation based on this approach allows patients to gain full benefit from NIV.

\section{P157 STAFF EDUCATION IMPROVES NON-INVASIVE VENTILATION OUTCOMES IN COPD PATIENTS PRESENTING WITH ACUTE HYPERCAPNOEIC RESPIRATORY FAILURE.}

doi:10.1136/thx.2010.151043.8

S Roychowdhury, O Kankam, C Jackson, D Sword, K Prasad, V Stark. The Ayr Hospital, NHS Ayrshire \& Arran, Ayr, UK

Introduction Non invasive ventilation (NIV) is safe and effective treatment for acute hypercapnoeic respiratory failure. National 